\title{
Entropy estimates for simple random fields
}

\author{
Forchhammer, Søren; Justesen, Jørn
}

Published in:

Proceedings of the IEEE International Symposium on Information Theory

Link to article, DOI:

10.1109/ISIT.1995.535764

Publication date:

1995

Document Version

Publisher's PDF, also known as Version of record

Link back to DTU Orbit

Citation (APA):

Forchhammer, S., \& Justesen, J. (1995). Entropy estimates for simple random fields. In Proceedings of the IEEE International Symposium on Information Theory (pp. 249-249). IEEE. https://doi.org/10.1109/ISIT.1995.535764

\section{General rights}

Copyright and moral rights for the publications made accessible in the public portal are retained by the authors and/or other copyright owners and it is a condition of accessing publications that users recognise and abide by the legal requirements associated with these rights.

- Users may download and print one copy of any publication from the public portal for the purpose of private study or research.

- You may not further distribute the material or use it for any profit-making activity or commercial gain

- You may freely distribute the URL identifying the publication in the public portal

If you believe that this document breaches copyright please contact us providing details, and we will remove access to the work immediately and investigate your claim. 


\title{
Entropy Estimates for Simple Random Fields
}

\author{
Søren Forchhammer and Jørn Justesen \\ Institute of Telecommunication, \\ Technical University of Denmark
}

\section{Introduction}

We consider the problem of determining the maximum entropy of a discrete random field on a lattice subject to certain local constraints on symbol configurations. The results are expected to be of interest in the analysis of digitized images and two dimensional codes. We shall present some examples of binary and ternary fields with simple constraints. Exact results on the entropies are known only in a few cases, but we shall present close bounds and estimates that are computationally efficient.

\section{Fields with Simple Constraints}

We consider random variables on a rectangular grid, $x(i, j)$. The lattice is defined by the set of neighbors associated with a given point. We shall not assume that the probability distribution is given, but the structure of the field will be specified in terms of a set of constraints on the values assumed by a particular variable and its neighbors. Constraints could be of one of the following (not necessarily distinct) types:

- the runs of pixels of a given color should satisfy a set of inequalities [1] [2]

- the field is a random tiling of the plane with certain pieces

- certain configurations of values are excluded

Since we are interested in estimates of the entropy which may be related to coding and data compression, we consider fields which are obviously stationary. The existence of solutions to the constraints should not be a problem, and boundary conditions should not be important.

Example 1: As a simple example we shall consider the following problem which is quite well-known: Consider a binary field on a rectangular lattice with the restriction that two neighbors, i.e. $x(i, j)$ and $x(i, j+1)$ or $x(i, j)$ and $x(i+1, j)$, cannot both have the value 1 . What is the largest possible entropy, or what is the number of solutions for an $N$ by $N$ segment of the lattice as a function of $N$ ? We estimate the entropy to be $H \approx 0.587891161775339$.

\section{Markov Chains}

As suggested in [1], the maximal entropy may be bounded by the entropy of a band of finite width, i.e. the variable $j$ is restricted to $0<j \leq m$. This entropy can be calculated as the maximal entropy of a finite state Markov chain, and from this approach we obtain an upper bound (with a suitable relaxation on the restrictions at the boundaries). This estimate converges slowly. For the problem of Example 1 we get $H<$ 0.5928 for $m=20$ imposing no restrictions at the boundaries. Constraining the probability of a 1 at the boundaries a tighter bound may be obtained. In some cases it is possible to derive a very accurate estimate from this sequence of values, $H_{m}$. The estimate given in Example 1 was obtained as $H_{m+1}-H_{m}$ with $m=16$.

Another type of estimate may be obtained from finite state causal models of the field. If the outcome of the process is generated one pixel at a time, and the probability distribution of $x\left(i^{\prime}, j^{\prime}\right)$ is assumed to depend on a finite past context $i<i^{\prime}$ or $i=i^{\prime}$ and $j<j^{\prime}$, then the entropy can be approximated by that of a finite Markov source. This approach gives some information about the properties of the field, but the model is only exact in a very simple case, which is discussed in the following section.

\section{Construction of Stationary Fields}

An actual construction of a random field with known entropy is interesting both for simulation purposes and as a method for establishing lower bounds. It would be very desirable to have random fields where rows and columns were described by simple Markov chains. Unfortunately this appears to be possible only in the case of the Pickard lattices [3]. This is also the only case where the causal model of the field becomes a simple finite state source.

Example 2: A Pickard field consistent with the constraint considered in Example 1 may be constructed such that each row or column is a Markov chain with $P(1)=1 / 5$. In this case the entropy may be found explicitly as $H=1 / 10+3 / 10 \log 3$ $=0.575 \ldots$ Actually a slightly larger value may be obtained by varying the transition probabilities.

Clearly the solution to the maximum entropy problem is always a Markov random field. However, in general such fields are hard to analyze. We shall consider a construction which has much greater flexibility than the Pickard field, but still allows detailed analysis:

Let rows $i$ and $i+1$ be generated by a Markov chain (or another unifilar finite state source), such that there is complete symmetry between the two rows. Their joint entropy can be easily calculated. The probability distribution may be extended to a stationary distribution on the entire plane by assuming that all pairs of rows have the same distribution, and that the probability of each row given the past depends on only the previous row. The entropy of a row given the previous row may, in some cases, be calculated from a hidden Markov source.

Example 3: For the constraint in Example 1, two successive rows may be generated by a symmetric 3 -state Markov chain with entropy $\mathrm{H}_{2}$. From this source it is possible to calculate the entropy of a single row $(i), H_{1}$, exactly. Whenever $x(i, j)=1$, the state of the source is known, and the distribution of zero runs can be calculated. We find the entropy of the process as $H=H_{2}-H_{1}$. The largest lower bound obtained in this way is 0.58783 .

\section{References}

[1] V. K. Wei and T. Etzion, "On two-dimensional run-length limited codes", IEEE Information Theory Workshop, Brazil, 1992.

[2] R. Burton and R. Pemantle, "Local characteristics, entropy and limit theorems for spanning trees and domino tilings via transfer-impedances", Ann. Prob., vol. 21, pp. 1329-71, 1993.

[3] D.K. Pickard, "A curious binary lattice process", J. Appl. Prob., vol. 14, pp. 717-731, 1977 